\title{
Elucidation of Selectivity Difference in the Diels-Alder Reactions of 6,6-Disubstituted Cyclohexa-2,4-dienone
}

\author{
Jun-Pyeong Jeong, Oh-Seuk Lee, and Kiyull Yang ${ }^{\star}$ \\ Department of Chemistry, Andong National University, Andong, Kyoungbuk 760-749, Korea \\ ${ }^{\dagger}$ Department of Chemistry: Education, Gyeongsang National Lniversity, Chinju 660-701, Korea \\ Received March 12, 2002
}

\begin{abstract}
Recently it was reported that cycloaddition of 6,6-disubstituted cyclohexa-2,4-dienone, 1 with cyclopentadiene gave solely the adduct of type I, while its reaction with 1,3-cyclohexadiene gave both II and III. Semiempirical MO calculations were done to elucidate the origin of the selectivity difference between the two dienes. Cycloaddition of 1 with cyclopentadiene is controlled thermodynamically to give only 1 -diene adduct by $\Delta G$ values of 10.6-20.3 kcal/mol, while its reaction with 1,3-cyclohexadiene does not show 1-diene/1-dienophile selectivity due to similar stabilities of the two adducts. Thenmodynamic parameters also show that endo adducts are more favourably formed in the cycloadditions of 1 with both cyclopentadiene and 1,3-cyclohexadiene, which coincides with experimental observations. Cope rearangements of $\varepsilon$ ndo adducts are another avenue to convert between 1-diene and 1-dienophile.
\end{abstract}

Keywords : Cycloaddition. Diels-Alder reaction. Cyclohexa-2,4-dienone. Semiempirical MO.

\section{Introduction}

Much interest has been focused on the pericyclic reactions of conjugated polyenes due to their potential for easy creation of complex carbocyclic systems. ${ }^{1,2} 2,4$-Cyclohexadienones are emerging as valuable intermediates in organic synthesis, ${ }^{3-6}$ because of their various chemical behavior. However, multiple modes of addition could be occurred during their cycloaddition with conjugated polyenes. ${ }^{\text {? }}$

In the syntheses of variously annulated bicyclo[2.2.2]octenones, cycloadditions of 6,6-disubstituted cyclohexa2,4-dienone 1 with cyclopentadiene gave only adduct of type I, while its reaction with 1,3-cyclohexadiene gave both II and III $^{8}$ (Scheme 1). Considering the structural similarity in cyclopentadiene and 1,3-cyclohexadiene, and that DielsAlder reaction is carried out more easily when dienophile has electron-withdrawing group, ${ }^{9}$ this observations are quite interesting.

It has been reported that the reactions of variously substituted cyclohexa-2,4-dienones with cyclopentadiene involve the intermediate formation of norbornene system, where cyclopentadiene behaves as diene, which then rear-

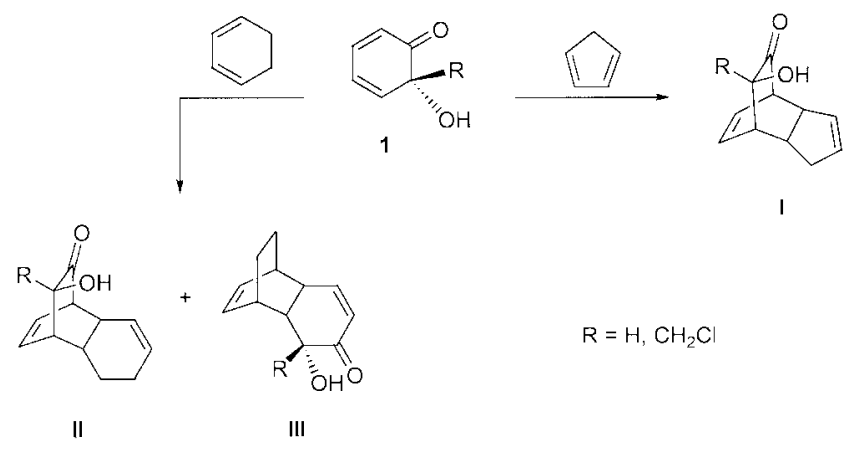

Scheme 1 ranges to give a less strained product, adduct of type $I .^{\text {sa }}$ However, it cannot be excluded that some of the adduct might have been formed directly by addition of the cyclohexadienone to a double bond of the cyclopentadiene.

On the other hand, Singh and coworkers have shown that cyclohexa-2,4-dienones behave as diene in the reaction with 1,3-cyclohexadiene, and that while the type III adducts failed to undergo the Cope rearrangement to type II adducts, the type II adducts smoothly rearranged to type III adducts. ${ }^{\text {s }}$ But they did not ruled out the possibility of a competitive reaction in which 1,3-cyclohexadiene behaves as diene.

In order to obtain a deeper insight into the origin of different behaviour of 1 in these reactions, we have performed semiempirical $\mathrm{PM}^{10}$ computations for these reactions and discussed the experimental results with thermodynamic parameters obtained.

\section{Computations}

All calculations were performed with PM3 method by using MOPAC $93,{ }^{11}$ and convergence criteria were increased by 100 times using keyword PRECISE. Transition states were located by using the eigenvector following procedure $(\mathrm{EF})^{12}$ and characterized by confirming the presence of only one negative eigenvalue in the Hessian matrix. In addition, intrinsic reaction coordinate (IRC) method $^{1 ?}$ was used to confirm both minima.

\section{Results and Discussion}

Frontier molecular orbitals were examined in the first place, in order to see if there is any aspect to make cyclopentadiene react only in one mode. PM3-optimized energy diagram of frontier molecular orbitals of $1\left(\mathrm{R}=\mathrm{CH}_{3}\right)$, cyclopentadiene, and 1,3-cyclohexadiene is shown in Figure 1. 


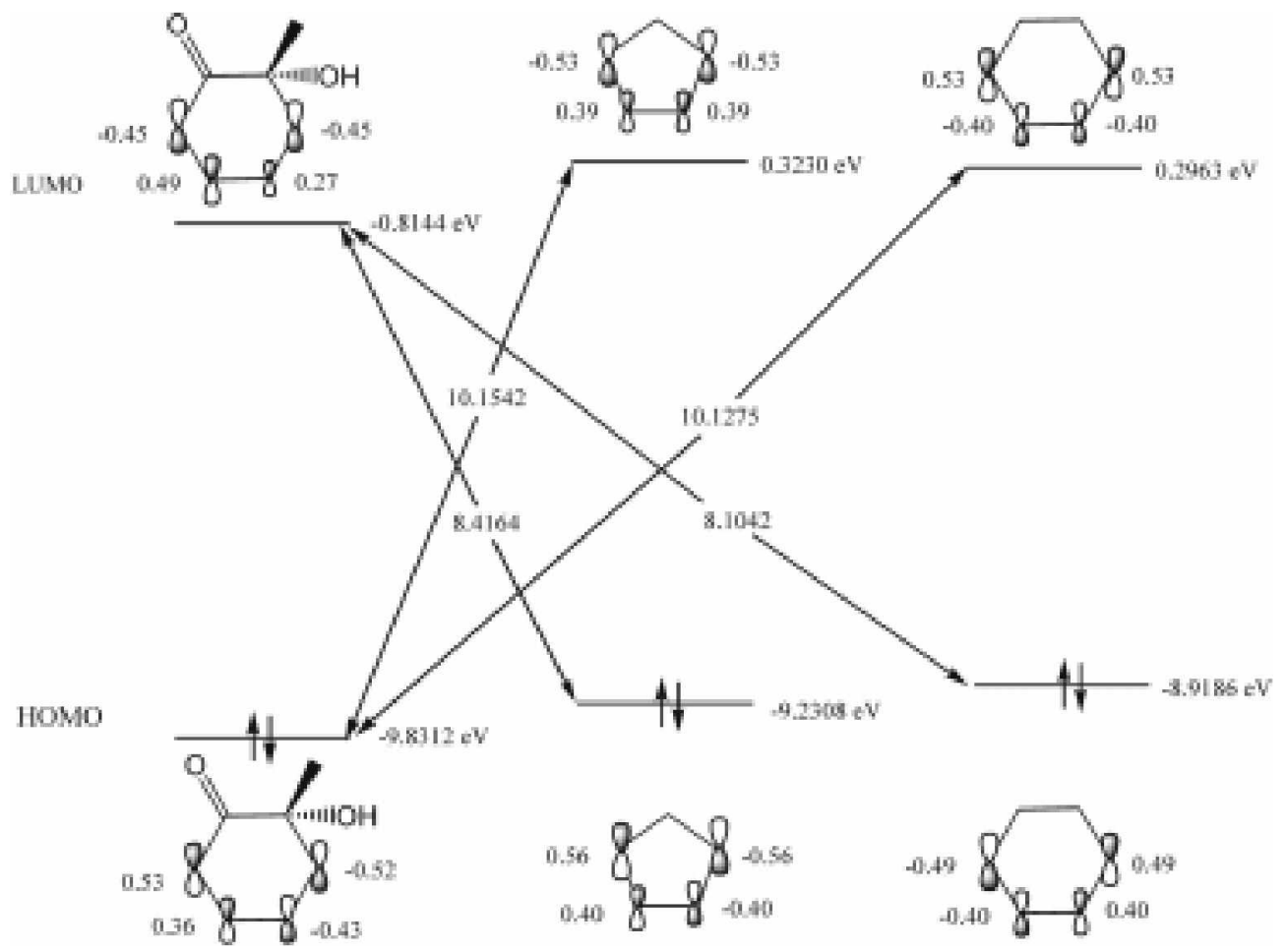

Figure 1. PM3-optimized energy diagram of frontier molecular orbitals of $1\left(\mathrm{R}=\mathrm{CH}_{3}\right)$. cyclopentadiene, and cyclohexadiene. Part of coefficients of the FMOs are depicted.

The interaction between LUMO of $1\left(\mathrm{R}=\mathrm{CH}_{3}\right)$ and HOMO of cyclopentadiene is more favourable than the inteaction between HOMO of $1\left(\mathrm{R}=\mathrm{CH}_{3}\right)$ and LUMO of cyclopentadiene in terms of energy by $1.74 \mathrm{eV}$, although both interactions are symmetry-allowed. Frontier molecular orbital interaction energy, $\Delta \mathrm{E}_{\mathrm{FvO}}$, for the cycloaddition between two molecules can be estimated by ${ }^{14}$

$\Delta E_{F H O}=$

$2 \gamma^{2}\left[\frac{\left(C_{A 1 H} C_{B 1 L}+C_{A 2 H} C_{B 2 L}\right)^{2}}{E_{A H}-E_{B L}}+\frac{\left(C_{A 1 L} C_{B 1 H}+C_{A 2 L} C_{B 2 H}\right)^{2}}{E_{B H}-E_{A L}}\right]$

where $\gamma$ is the resonance integral of the two interacting lobes at each of the reaction sites, $C$ and $E$ refer to particular eigenvector coefficient and eigenvalue, respectively. Subscripts A and B refer to the two reacting molecules, 1 and 2 refer to the two reaction sites, and $\mathrm{H}$ and $\mathrm{L}$ refer to HOMO and LUMO. $\triangle \mathrm{E}_{\mathrm{FMO}}$ values were compared for the two reaction modes in the interaction between $1\left(\mathrm{R}=\mathrm{CH}_{3}\right)$ and cyclopentadiene. The reaction of $\mathbf{1}\left(\mathrm{R}=\mathrm{CH}_{3}\right)$ as dienophile $\left(-0.11 \gamma^{2} \mathrm{eV}\right)$ is slightly more favourable than that as diene $\left(-0.092 \gamma^{2} \mathrm{eV}\right)$, although both reactions are symmetry-allowed. Energy is more favourable for the interaction between LUMO of $1\left(\mathrm{R}=\mathrm{CH}_{3}\right)$ and HOMO of 1,3-cyclohexadiene, as is the same aspect in the reaction of $1\left(\mathrm{R}=\mathrm{CH}_{3}\right)$ with cyclopentadiene, by $2.02 \mathrm{eV}$. Again, the reaction of $1\left(\mathrm{R}=\mathrm{CH}_{3}\right)$ as dienophile $\left(-0.10 \gamma^{2} \mathrm{eV}\right)$ is slightly more favourable than that as diene $\left(-0.094 \gamma^{2} \mathrm{eV}\right)$ as regards to frontier molecular orbital interaction energy. Analysis of FMO did not give the grounds for the selectivity of the reaction of 1 with cyclo- pentadiene.

We have sought all the stationary points for possible reaction paths in the cycloadditions of 1 with cyclopentadiene and 1,3-cyclohexadiene.

Cycloadditions with cyclopentadiene. Scheme 2 shows all feasible structures resulted from cycloadditions of 1 with cyclopentadiene. While $\mathbf{2}, \mathbf{2 a}, \mathbf{2} \mathbf{b}$, and $\mathbf{2 c}$ could be expected when cyclopentadiene reacts as dienophile, $\mathbf{3}, \mathbf{3 a}, \mathbf{3 b}$, and $\mathbf{3 c}$ could be obtained when it reacts as diene. All structures could have a pair of cis junctions, i.e., endo and exo additions.

Stereoisomers of each adduct of 1 in Scheme 2 were not considered, because they did not show stability difference. Computations were performed for $\mathrm{R}=\mathrm{H}, \mathrm{CH}_{3}$, and $\mathrm{CH}_{2} \mathrm{Cl}$ of
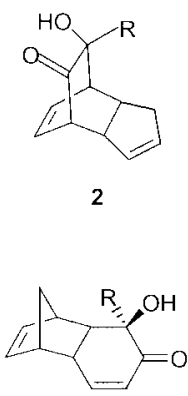

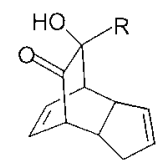

$2 a$

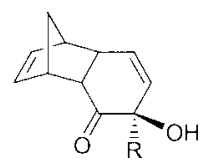

3a

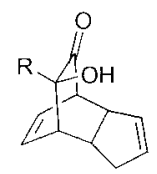

2b

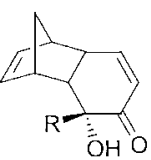

$3 b$

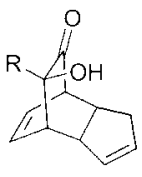

2c

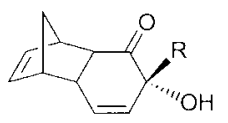

$3 c$
$\mathrm{R}=\mathrm{H}, \mathrm{CH}_{3}, \mathrm{CH}_{2} \mathrm{Cl}$

Scheme 2 
Table 1. PM3-calculated thermodynamic parametersi2 for the Diels-Alder reactions of 1 with cyclopentadiene in $\mathrm{kcal} / \mathrm{mol}$ at $298 \mathrm{~K}$

\begin{tabular}{|c|c|c|c|c|c|c|c|c|c|}
\hline \multicolumn{2}{|c|}{ Compounds } & \multirow{2}{*}{$\frac{\Delta \mathrm{H}_{\mathrm{i}}}{-47.2}$} & \multirow{2}{*}{$\frac{S^{b}}{78.1}$} & \multirow[t]{2}{*}{$\Delta \mathrm{H}$} & \multirow[t]{2}{*}{$\Delta S^{h}$} & \multirow[t]{2}{*}{$\Delta G$} & \multirow[t]{2}{*}{$\Delta \mathrm{H}^{*}$} & \multirow[t]{2}{*}{$\Delta \mathrm{S}^{\neq b}$} & \multirow[t]{2}{*}{$\Delta G^{\ddagger}$} \\
\hline 1 & $\mathrm{R}=\mathrm{H}$ & & & & & & & & \\
\hline & $\mathrm{CH}_{3}$ & -53.0 & 84.3 & & & & & & \\
\hline & $\mathrm{CH}_{2} \mathrm{Cl}$ & -54.0 & 86.4 & & & & & & \\
\hline \multicolumn{2}{|c|}{ cyclopentadiene } & 31.7 & 65.3 & & & & & & \\
\hline \multirow[t]{3}{*}{2 -ento } & $\mathrm{R}=\mathrm{H}$ & -45.2 & 92.9 & -29.7 & -50.5 & -14.6 & 36.7 & -49.6 & 51.5 \\
\hline & $\mathrm{CH}_{3}$ & -50.8 & 97.9 & -29.5 & -51.7 & -14.1 & 37.2 & -51.5 & 52.6 \\
\hline & $\mathrm{CH}_{2} \mathrm{Cl}$ & -52.2 & 100.3 & -29.9 & -51.4 & -14.6 & 36.6 & -47.9 & 50.9 \\
\hline \multirow[t]{3}{*}{$2-e x o$} & $\mathrm{R}=\mathrm{H}$ & -45.3 & 91.8 & -29.8 & -51.6 & -14.4 & 37.7 & -47.5 & 51.9 \\
\hline & $\mathrm{CH}_{3}$ & -47.4 & 96.5 & -26.1 & -53.1 & -10.3 & 42.4 & -48.6 & 56.9 \\
\hline & $\mathrm{CH}_{2} \mathrm{Cl}$ & -49.2 & 99.1 & -26.9 & -52.6 & -11.2 & 41.5 & -48.5 & 56.0 \\
\hline \multirow[t]{3}{*}{$2 \mathbf{a}-e n d o$} & $\mathrm{R}=\mathrm{H}$ & -45.2 & 92.9 & -29.7 & -50.5 & -14.6 & 36.9 & -49.4 & 51.6 \\
\hline & $\mathrm{CH}_{\mathbf{3}}$ & -50.9 & 97.9 & -29.6 & -51.7 & -14.2 & 37.3 & -47.9 & 51.6 \\
\hline & $\mathrm{CH}_{2} \mathrm{Cl}$ & -51.8 & 100.4 & -29.5 & -51.3 & -14.2 & 37.5 & -47.9 & 51.8 \\
\hline \multirow[t]{3}{*}{$2 \mathbf{a}-x_{0}$} & $\mathrm{R}=\mathrm{H}$ & -45.2 & 92.4 & -29.7 & -510 & -14.5 & 37.9 & -47.0 & 51.9 \\
\hline & $\mathrm{CH}_{3}$ & -47.8 & 98.1 & -26.5 & -51.5 & -11.1 & 42.1 & -47.7 & 56.3 \\
\hline & $\mathrm{CH}_{2} \mathrm{Cl}$ & -49.4 & 99.8 & -27.1 & -51.9 & -11.6 & 41.5 & -48.0 & 55.8 \\
\hline \multirow[t]{3}{*}{$2 \mathbf{b}-\epsilon) t d o$} & $\mathrm{R}=\mathrm{H}$ & -45.2 & 92.7 & -29.7 & -50.7 & -14.6 & 35.4 & -46.8 & 49.4 \\
\hline & $\mathrm{CH}_{3}$ & -50.7 & 98.7 & -29.4 & -50.9 & -14.2 & 36.0 & -47.0 & 50.0 \\
\hline & $\mathrm{CH}_{2} \mathrm{Cl}$ & -52.1 & 100.6 & -29.8 & -51.1 & -14.6 & 35.8 & -47.3 & 49.9 \\
\hline \multirow[t]{3}{*}{$2 \mathbf{b}-\mathrm{exo}$} & $\mathrm{R}=\mathrm{H}$ & -43.0 & 92.5 & -27.5 & -50.9 & -12.3 & 37.8 & -48.7 & 52.3 \\
\hline & $\mathrm{CH}_{3}$ & -47.6 & 98.0 & -26.3 & -51.6 & -10.9 & 39.9 & -48.9 & 54.5 \\
\hline & $\mathrm{CH}_{2} \mathrm{Cl}$ & -49.0 & 100.0 & -26.7 & -51.7 & -11.3 & 39.6 & -48.8 & 54.1 \\
\hline \multirow[t]{3}{*}{$2 \mathrm{c}-\mathrm{e} n d o$} & $\mathrm{R}=\mathrm{H}$ & -45.3 & 92.7 & -29.8 & -50.7 & -14.7 & 35.7 & -46.9 & 49.7 \\
\hline & $\mathrm{CH}_{3}$ & -50.7 & 98.7 & -29.4 & -50.9 & -14.2 & 36.2 & -47.0 & 50.2 \\
\hline & $\mathrm{CH}_{2} \mathrm{Cl}$ & -52.1 & 100.6 & -29.8 & -51.1 & -14.6 & 36.0 & -47.3 & 50.1 \\
\hline \multirow[t]{3}{*}{$2 \mathrm{c}-\mathrm{e} \times \mathrm{O}$} & $\mathrm{R}=\mathrm{H}$ & -43.2 & 92.6 & -27.7 & -50.8 & -12.6 & 36.9 & -48.5 & 51.4 \\
\hline & $\mathrm{CH}_{3}$ & -48.0 & 98.2 & -26.7 & -51.4 & -11.4 & 40.1 & -47.3 & 54.2 \\
\hline & $\mathrm{CH}_{2} \mathrm{Cl}$ & -49.6 & 100.3 & -27.3 & -51.4 & -12.0 & 39.9 & -51.1 & 55.1 \\
\hline 3-endo & $\mathrm{R}=\mathrm{H}$ & -30.4 & 87.6 & -14.9 & -55.8 & 1.7 & 37.7 & -50.6 & 52.8 \\
\hline & $\mathrm{CH}_{3}$ & -31.7 & 93.8 & -10.4 & -55.8 & 6.2 & 41.1 & -51.7 & 56.5 \\
\hline & $\mathrm{CH}_{2} \mathrm{Cl}$ & -33.4 & 100.2 & -11.1 & -51.5 & 4.3 & 40.6 & -51.3 & 55.9 \\
\hline $3-e x o$ & $\mathrm{R}=\mathrm{H}$ & -30.7 & 89.2 & -15.2 & -54.2 & 1.0 & 38.0 & -52.1 & 53.5 \\
\hline & $\mathrm{CH}_{3}$ & -34.4 & 95.3 & -13.1 & -54.3 & 3.1 & 38.6 & -50.9 & 53.8 \\
\hline & $\mathrm{CH}_{2} \mathrm{Cl}$ & -35.9 & 97.1 & -13.6 & -54.6 & 2.7 & 38.1 & -51.1 & 53.3 \\
\hline $\mathbf{3} \mathbf{a}-e n d o$ & $\mathrm{R}=\mathrm{H}$ & -29.8 & 89.9 & -14.3 & -53.5 & 1.7 & 36.0 & -50.3 & 51.0 \\
\hline & $\mathrm{CH}_{3}$ & -35.1 & 95.3 & -13.8 & -54.3 & 2.4 & 36.2 & -48.6 & 50.7 \\
\hline & $\mathrm{CH}_{2} \mathrm{Cl}$ & -37.6 & 97.4 & -15.3 & -54.3 & 0.9 & 36.3 & -50.9 & 51.5 \\
\hline 3a-exo & $\mathrm{R}=\mathrm{H}$ & -32.9 & 89.1 & -17.4 & -54.3 & -1.2 & 36.6 & -50.4 & 51.6 \\
\hline & $\mathrm{CH}_{3}$ & -37.2 & 95.5 & -15.9 & -54.1 & 0.2 & 36.2 & -50.9 & 51.4 \\
\hline & $\mathrm{CH}_{2} \mathrm{Cl}$ & -38.9 & 96.9 & -16.6 & -54.8 & -0.3 & 36.0 & -51.4 & 51.3 \\
\hline $\mathbf{3 b}-\epsilon \| t d o$ & $\mathrm{R}=\mathrm{H}$ & -29.4 & 88.5 & -13.9 & -54.9 & 2.5 & 37.1 & -51.4 & 52.4 \\
\hline & $\mathrm{CH}_{3}$ & -33.0 & 95.1 & -11.7 & -54.5 & 4.5 & 39.0 & -50.9 & 54.2 \\
\hline & $\mathrm{CH}_{2} \mathrm{Cl}$ & -34.4 & 97.0 & -12.1 & -54.7 & 4.2 & 38.6 & -51.0 & 53.8 \\
\hline $3 \mathbf{b}$-exo & $\mathrm{R}=\mathrm{H}$ & -32.5 & 87.9 & -17.0 & -55.5 & -0.5 & 37.0 & -47.5 & 51.2 \\
\hline & $\mathrm{CH}_{3}$ & -34.9 & 97.3 & -13.6 & -52.3 & 20 & 40.5 & -50.5 & 55.6 \\
\hline & $\mathrm{CH}_{2} \mathrm{Cl}$ & -36.3 & 99.7 & -14.0 & -52.0 & 1.5 & 40.3 & -45.8 & 54.0 \\
\hline $\mathbf{3 c}-\varepsilon \| t d o$ & $\mathrm{R}=\mathrm{H}$ & -31.6 & 89.0 & -16.1 & -54.4 & 0.1 & 36.4 & -51.8 & 51.8 \\
\hline & $\mathrm{CH}_{3}$ & -35.9 & 95.5 & -14.6 & -54.1 & 1.5 & 37.2 & -50.7 & 52.3 \\
\hline & $\mathrm{CH}_{2} \mathrm{Cl}$ & -37.6 & 96.8 & -15.3 & -54.9 & 1.1 & 36.9 & -51.3 & 52.2 \\
\hline $3 c-e \times o$ & $\mathrm{R}=\mathrm{H}$ & -32.9 & 91.6 & -17.4 & -51.8 & -2.0 & 34.6 & -47.4 & 48.7 \\
\hline & $\mathrm{CH}_{3}$ & -37.6 & 95.4 & -16.3 & -54.2 & -0.1 & 34.9 & -48.4 & 49.3 \\
\hline & $\mathrm{CH}_{2} \mathrm{Cl}$ & -38.8 & 97.5 & -16.5 & -54.2 & -0.3 & 34.9 & -48.5 & 49.4 \\
\hline
\end{tabular}

$\Delta \mathrm{H}_{f}=$ Heat of formation. $\Delta \mathrm{H}, \Delta \mathrm{S}$, and $\Delta \mathrm{G}$ refer to reaction enthalpy, reaction entropy, and Gibbs free energy of reaction, respectively, $\Delta \mathrm{H}^{\neq}, \Delta \mathrm{S}^{\vec{*}}$, and $\Delta G^{x}$ refer to activation enthalpy, activation entropy, and Gibbs free energy of activation, respectively. ${ }^{b} \mathrm{cal}$ ( $\left.\mathrm{K} \cdot \mathrm{mol}\right)$. 
Table 2. Comparison of thermodynamic parameters between 1 diene and 1-dienophile for the Diels-Alder reaction of $\mathbf{1}$ with cyclopentadiene in $\mathrm{kcal} / \mathrm{mol}$

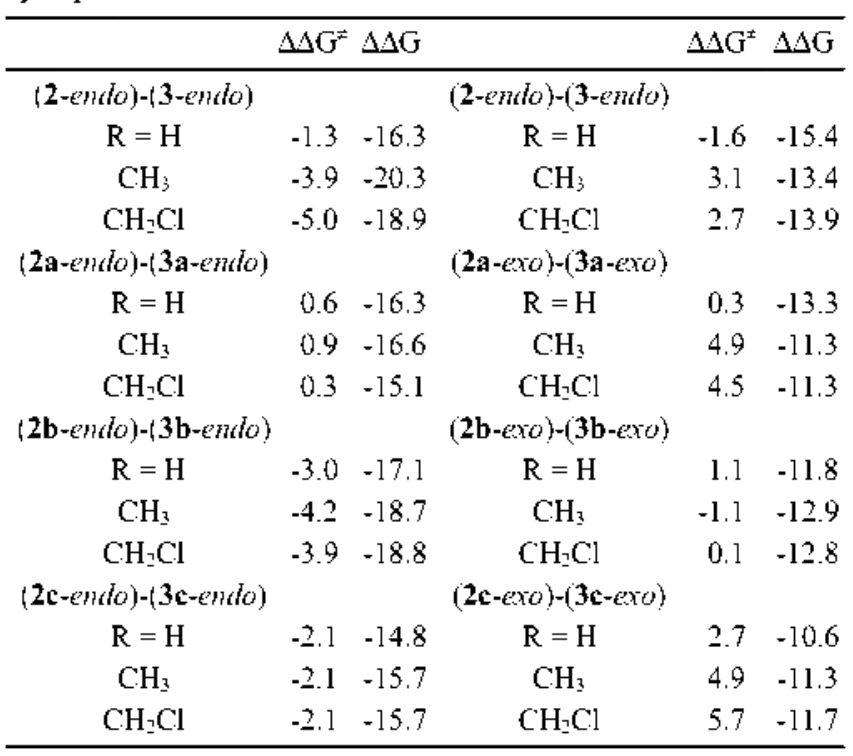

1 to see if substituent effect exist.

Table 1 shows thermodynamic parameters for the cycloaddition reaction of 1 with cyclopentadiene at $298 \mathrm{~K}$.

Themodynamic parameters of endo and exo adduct were compared to see if there are stability differences that could result in endo/exo selectivity; the difference of Gibbs free energy of reaction, $\Delta \Delta \mathrm{G}$ (endo-exo) and the difference of Gibbs free energy of activation, $\Delta \Delta \mathrm{G}^{\ddagger}$ (endo-exo) reveal that adducts which arise from the reaction of $\mathbf{1}$ as diene (1-diene) are all favourable for endo addition by $0.1-3.8 \mathrm{kcal} / \mathrm{mol}$ and $0.3-5.1 \mathrm{kcal} / \mathrm{mol}$, respectively. Adducts which arise from the reaction of 1 as dienophile (1-dienophile) are all favourable for exo addition by Gibbs free energies of reaction of 0.7-3.1 $\mathrm{kcal} / \mathrm{mol}$, while Gibbs free energy of activation do not show consistency.

Both 1 and cyclopentadiene could function as diene in Diels-Alder cycloaddition, hence it is necessary to find 1diene/1-dienophile selectivity. Themodynamic parameters of 1-diene and 1-dienophile were compared to see if there are stability differences that could result in 1-diene/1-dienophile selectivity (Table 2)

The differences of Gibbs free energy of reaction for all endo adducts and exo adducts are favourable for 1-diene cycloaddition by $10.6-20.3 \mathrm{kcal} / \mathrm{mol}$, while the differences of Gibbs free energy of activation, which range from 0.1 $\mathrm{kcal} / \mathrm{mol}$ to $5.7 \mathrm{kcal} / \mathrm{mol}$, do not show consistency. This reveals that 1-diene/1-dienophile selectivity for the cycloaddition of 1 with cyclopentadiene was thermodynamically controlled. Potential energy diagrams for the cycloadditions of 1 with cyclopentadiene are shown in Figure 2.

It is interesting that 1-diene endo adducts can be formed through Cope rearrangement of 1 -dienophile endo adducts and vice versa. Table 3 shows thermodynamic parameters for the Cope rearrangement of endo adducts of 1 with cyclopentadiene.

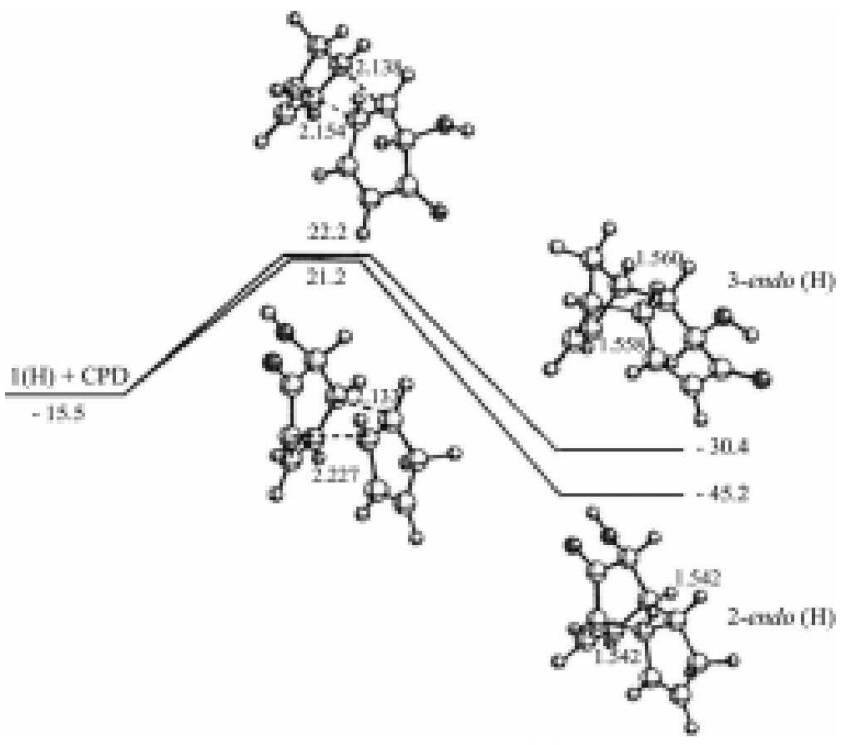

Figure 2. Potential energy $\left(\Delta \mathrm{H}_{i}\right)$ diagram for the cycloadditions in compound $1(\mathrm{R}=\mathrm{H})$ with cyclopentadiene (CPD) calculated by the PM3 Hamiltonian. Energies and interatomic distances are given in kcalmol and angstrom, respectively.

Table 3. PM3-calculated thennodynamic parameters for the Cope rearrangement of the adducts of 1 with cyclopentadiene in $\mathrm{kcal} / \mathrm{mol}$ at $298 \mathrm{~K}$

\begin{tabular}{ccccccc}
\hline Reactions & $\Delta \mathrm{H}$ & $\Delta \mathrm{S}^{a}$ & $\Delta \mathrm{G}$ & $\Delta \mathrm{H}^{\mp}$ & $\Delta \mathrm{S}^{\mp a}$ & $\Delta \mathrm{G}^{x}$ \\
\hline 3-endo to 2-endo & & & & & \\
$\mathrm{R}=\mathrm{H}$ & -14.8 & 5.3 & -16.4 & 51.9 & 4.3 & 50.6 \\
$\mathrm{CH}_{3}$ & -19.1 & 4.1 & -20.3 & 48.0 & 2.8 & 47.2 \\
$\mathrm{CH}_{2} \mathrm{Cl}$ & -18.8 & 0.1 & -18.8 & 48.9 & -0.5 & 49.0 \\
3a-endo to 2a-endo & & & & & \\
$\mathrm{R}=\mathrm{H}$ & -15.4 & 3.0 & -16.3 & 52.6 & 1.5 & 52.2 \\
$\mathrm{CH}_{3}$ & -15.8 & 2.6 & -16.6 & 53.4 & 1.0 & 53.1 \\
$\mathrm{CH}_{2} \mathrm{Cl}$ & -14.2 & 3.0 & -15.1 & 54.8 & 1.1 & 54.5 \\
3b-endo to $\mathbf{2 b}-e n d o$ & & & & & \\
$\mathrm{R}=\mathrm{H}$ & -15.8 & 4.2 & -17.1 & 49.6 & 3.6 & 48.5 \\
$\mathrm{CH}$ & -17.7 & 3.6 & -18.8 & 47.8 & 2.5 & 47.1 \\
$\mathrm{CH}$ & -17.7 & 3.6 & -18.8 & 47.7 & 2.6 & 46.9 \\
3c-endo to 2c-endo & & & & & \\
$\mathrm{R}=\mathrm{H}_{3}$ & -13.7 & 3.7 & -14.8 & 56.0 & 2.3 & 55.3 \\
$\mathrm{CH}_{3}$ & -14.8 & 3.2 & -15.8 & 54.6 & 1.9 & 54.0 \\
$\mathrm{CH}_{2} \mathrm{Cl}$ & -14.5 & 3.8 & -15.6 & 54.9 & 2.5 & 54.2 \\
\hline
\end{tabular}

"cal:(K-mol)

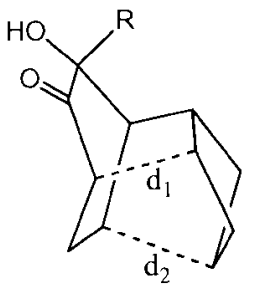

$\mathrm{TS}_{2-3}$

Gibbs free energies of activation and Gibbs free energies of reaction for the Cope rearrangement of 1-dienophile endo adducts are in the range of 46.9 to $55.3 \mathrm{kcal} / \mathrm{mol}$ and -14.8 to 
$-20.3 \mathrm{kcal} / \mathrm{mol}$, respectively. The values of Gibbs free energy of activation could be compared with those for the retro Diels-Alder reaction, which are in the range of 63.9 to 67.5 $\mathrm{kcal} / \mathrm{mol}$ for 1-diene adducts and 48.3 to $53.6 \mathrm{kcal} / \mathrm{mol}$ for 1 dienophile adducts. $d_{1}$ and $d_{2}$ values at ground state vary from $c a .1 .55 \AA$ of normal $\mathrm{C}$-C single bond distance to the range of $3.62 \AA-4.09 \AA$, and those values at transition state are in the range of $2.24 \AA-2.29 \AA$.

The experimental results for cycloadditions of 1 with cyclopentadiene, which gave only 1-diene adduct without giving 1-dienophile, might be explained with more favourable Gibbs free energies of reaction by $10.6-20.3 \mathrm{kcal} / \mathrm{mol}$, that is, 1-diene/1-dienophile selectivity for the cycloaddition of 1 with cyclopentadiene was thermodynamically controlled at reversible reaction conditions. Considering the values of Gibbs free energy of activation for the Cope rearrangement of 1-dienophile endo adducts and those for the retro Diels-Alder reaction of 1-dienophile adducts, Cope rearrangement of 1-dienophile endo adducts is another way of converting of 1-dienophile endo adduct to 1-diene endo adduct. Besides, endolexo selectivities of 1-diene adducts are all favourable for endo adducts in terms of both Gibbs free energy of activation and Gibbs free energy of reaction. These results reveal that $\mathbf{1}$-diene endo adducts are most favourably formed in the cycloadditions of 1 with cyclo-

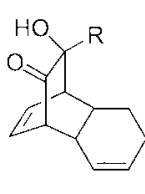

4

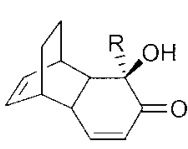

5

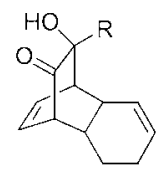

$4 a$

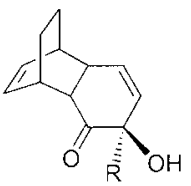

$5 a$

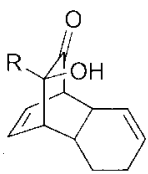

4b

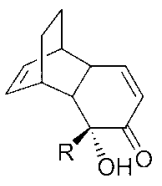

$5 b$

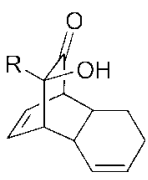

4c

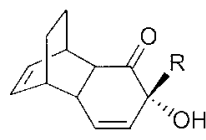

$5 c$

$$
\mathrm{R}=\mathrm{H}, \mathrm{CH}_{3}, \mathrm{CH}_{2} \mathrm{Cl}
$$

Scheme 3

pentadiene, which coincide with experimental observations. ${ }^{5.48 \mathrm{a}}$

Cycloadditions with 1,3-cyclohexadiene. Scheme 3 shows all feasible structures resulted from cycloadditions of 1 with 1,3-cyclohexadiene. While $\mathbf{4}, \mathbf{4 a}, \mathbf{4 b}$, and $\mathbf{4 c}$ could be expected when 1,3-cyclohexadiene reacts as dienophile, $\mathbf{5}$, $5 \mathbf{a}, \mathbf{5 b}$, and $\mathbf{5 c}$ could be obtained when it reacts as diene. All structures could have a pair of $c i s$ junctions, i.e., endo and exo additions.

Table 4 shows thernodynamic parameters for the cyclo-

Table 4. PM3-calculated thermodynamic parameters ${ }^{i 2}$ for the Diels-Alder reactions of 1 with 1,3-cyclohexadiene in kcal $/ \mathrm{mol}$ at $298 \mathrm{~K}$

\begin{tabular}{|c|c|c|c|c|c|c|c|c|c|}
\hline \multicolumn{2}{|c|}{ Compounds } & \multirow{2}{*}{$\frac{\Delta \mathrm{H}_{\mathrm{f}}}{-47.2}$} & \multirow{2}{*}{$\frac{S^{i}}{78.1}$} & \multirow[t]{2}{*}{$\Delta \mathrm{H}$} & \multirow[t]{2}{*}{$\Delta \mathrm{S}^{i}$} & \multirow[t]{2}{*}{$\Delta \mathrm{G}$} & \multirow[t]{2}{*}{$\Delta \mathrm{H}^{\mp}$} & \multirow[t]{2}{*}{$\Delta \mathrm{S}^{ \pm \dot{2}}$} & \multirow[t]{2}{*}{$\Delta \mathrm{G}^{\prime}$} \\
\hline 1 & $\mathrm{R}=\mathrm{H}$ & & & & & & & & \\
\hline & $\mathrm{CH}_{3}$ & -53.0 & 84.3 & & & & & & \\
\hline & $\mathrm{CH}_{2} \mathrm{Cl}$ & -54.0 & 86.4 & & & & & & \\
\hline cyclohex & & 20.3 & 72.0 & & & & & & \\
\hline \multirow[t]{3}{*}{ 4-entdo } & $\mathrm{R}=\mathrm{H}$ & -51.0 & 97.7 & -24.1 & -52.4 & -8.5 & 39.0 & -54.6 & 55.3 \\
\hline & $\mathrm{CH}_{3}$ & -56.8 & 102.6 & -24.1 & -53.7 & -8.1 & 39.3 & -56.5 & 56.1 \\
\hline & $\mathrm{CH}_{2} \mathrm{Cl}$ & -57.7 & 105.2 & -24.0 & -53.2 & -8.1 & 39.5 & -53.0 & 55.3 \\
\hline \multirow[t]{3}{*}{$4-e x o$} & $\mathrm{R}=\mathrm{H}$ & -51.4 & 96.9 & -24.5 & -53.2 & -8.6 & 39.7 & -52.4 & 55.3 \\
\hline & $\mathrm{CH}_{3}$ & -53.2 & 98.0 & -20.5 & -58.3 & -3.1 & 44.8 & -53.9 & 60.9 \\
\hline & $\mathrm{CH}_{2} \mathrm{Cl}$ & -54.4 & 103.8 & -20.7 & -54.6 & -4.4 & 44.8 & -50.4 & 59.8 \\
\hline \multirow[t]{2}{*}{$4 \mathbf{a}-t) d o$} & $\mathrm{R}=\mathrm{H}$ & -50.8 & 97.8 & -23.9 & -52.3 & -8.3 & 38.5 & -57.7 & 55.7 \\
\hline & $\mathrm{CH}_{3}$ & -56.7 & 102.7 & -24.0 & -53.6 & -8.0 & 39.4 & -53.0 & 55.2 \\
\hline \multirow[t]{3}{*}{$4 a-e x o$} & $\mathrm{R}=\mathrm{H}$ & -51.2 & 97.5 & -24.3 & -52.6 & -8.6 & 40.3 & -520 & 55.8 \\
\hline & $\mathrm{CH}_{3}$ & -54.9 & 102.7 & -22.2 & -53.6 & -6.2 & 43.8 & -49.4 & 58.5 \\
\hline & $\mathrm{CH}_{2} \mathrm{Cl}$ & -57.1 & 103.3 & -23.4 & -55.1 & -7.0 & 43.4 & -50.9 & 58.6 \\
\hline \multirow[t]{3}{*}{$4 \mathbf{b}-e n d o$} & $\mathrm{R}=\mathrm{H}$ & -50.9 & 97.5 & -24.0 & -52.6 & -8.3 & 37.9 & -51.9 & 53.4 \\
\hline & $\mathrm{CH}_{3}$ & -56.5 & 103.4 & -23.8 & -52.9 & -8.0 & 38.5 & -55.5 & 55.0 \\
\hline & $\mathrm{CH}_{2} \mathrm{Cl}$ & -57.8 & 105.4 & -24.1 & -53.0 & -8.3 & 38.3 & -52.5 & 54.0 \\
\hline \multirow[t]{3}{*}{$4 b-e x o$} & $\mathrm{R}=\mathrm{H}$ & -48.8 & 95.8 & -21.9 & -54.3 & -5.7 & 41.1 & -54.0 & 57.2 \\
\hline & $\mathrm{CH}_{3}$ & -54.1 & 101.8 & -21.4 & -54.5 & -5.2 & 41.9 & -53.9 & 58.0 \\
\hline & $\mathrm{CH}_{2} \mathrm{Cl}$ & -55.4 & 103.9 & -21.7 & -54.5 & -5.5 & 41.7 & -54.2 & 57.9 \\
\hline \multirow[t]{3}{*}{$4 c-\varepsilon n d o$} & $\mathrm{R}=\mathrm{H}$ & -51.0 & 97.4 & -24.1 & -52.7 & -8.4 & 37.8 & -51.9 & 53.3 \\
\hline & $\mathrm{CH}_{3}$ & -56.4 & 103.5 & -23.7 & -52.8 & -8.0 & 38.4 & -55.5 & 54.9 \\
\hline & $\mathrm{CH}_{2} \mathrm{Cl}$ & -57.8 & 105.4 & -24.1 & -53.0 & -8.3 & 38.2 & -52.6 & 53.9 \\
\hline $4 c-e \times 0$ & $\mathrm{R}=\mathrm{H}$ & -49.8 & 97.4 & -22.9 & -52.7 & -7.2 & 39.6 & -53.2 & 55.5 \\
\hline
\end{tabular}


Table 4. Continued

\begin{tabular}{|c|c|c|c|c|c|c|c|c|c|}
\hline \multicolumn{2}{|c|}{ Compounds } & \multirow{2}{*}{$\frac{\Delta \mathrm{H}_{\mathrm{f}}}{-51.2}$} & & \multirow{2}{*}{$\frac{\Delta H}{-24.3}$} & \multirow{2}{*}{$\frac{\Delta \mathrm{S}^{h}}{-57.0}$} & \multirow{2}{*}{$\frac{\Delta G}{-7.3}$} & \multirow{2}{*}{$\frac{\Delta H^{*}}{38.6}$} & \multirow{2}{*}{$\frac{\Delta S^{\neq h}}{-54.4}$} & \multirow{2}{*}{$\frac{\Delta G^{\ddagger}}{54.8}$} \\
\hline 5-ento & $\mathrm{R}=\mathrm{H}$ & & & & & & & & \\
\hline & $\mathrm{CH}_{3}$ & -52.2 & 98.5 & -19.5 & -57.8 & -2.3 & 42.7 & -54.4 & 58.9 \\
\hline & $\mathrm{CH}_{2} \mathrm{Cl}$ & -54.1 & 100.2 & -20.4 & -58.2 & -3.0 & 40.6 & -52.7 & 56.3 \\
\hline \multirow[t]{3}{*}{ 5-exo } & $\mathrm{R}=\mathrm{H}$ & -48.6 & 92.7 & -21.7 & -57.4 & -4.6 & 40.1 & -53.6 & 56.1 \\
\hline & $\mathrm{CH}_{3}$ & -51.9 & 98.5 & -19.2 & -57.8 & -2.0 & 42.1 & -53.5 & 58.1 \\
\hline & $\mathrm{CH}_{2} \mathrm{Cl}$ & -53.5 & 101.1 & -19.8 & -57.3 & -2.7 & 41.7 & -53.7 & 57.7 \\
\hline \multirow[t]{3}{*}{$5 \mathbf{a}-e n d o$} & $\mathrm{R}=\mathrm{H}$ & -51.3 & 93.2 & -24.4 & -56.9 & -7.4 & 36.3 & -50.9 & 51.5 \\
\hline & $\mathrm{CH}_{3}$ & -55.1 & 99.5 & -22.4 & -56.8 & -5.5 & 36.8 & -49.5 & 51.6 \\
\hline & $\mathrm{CH}_{2} \mathrm{Cl}$ & -57.2 & 101.5 & -23.5 & -56.9 & -6.5 & 36.8 & -49.4 & 51.5 \\
\hline \multirow[t]{3}{*}{$5 a-e \times o$} & $\mathrm{R}=\mathrm{H}$ & -50.9 & 94.0 & -24.0 & -56.1 & -7.3 & 38.2 & -54.3 & 54.4 \\
\hline & $\mathrm{CH}_{3}$ & -55.3 & 99.6 & -22.6 & -56.7 & -5.7 & 39.2 & -53.4 & 55.1 \\
\hline & $\mathrm{CH}_{2} \mathrm{Cl}$ & -56.9 & 100.9 & -23.2 & -57.5 & -6.1 & 39.1 & -53.9 & 55.2 \\
\hline \multirow[t]{3}{*}{$\left.\mathbf{5 b}-e^{\prime}\right)$} & $\mathrm{R}=\mathrm{H}$ & -49.1 & 94.0 & -22.2 & -56.1 & -5.5 & 38.5 & -54.9 & 54.9 \\
\hline & $\mathrm{CH}_{3}$ & -52.3 & 99.6 & -19.6 & -56.7 & -2.7 & 40.6 & -54.7 & 56.9 \\
\hline & $\mathrm{CH}_{2} \mathrm{Cl}$ & -53.8 & 101.3 & -20.1 & -57.1 & -3.1 & 40.1 & -51.7 & 55.5 \\
\hline \multirow[t]{3}{*}{$5 \mathbf{b}-\mathbf{e} \times$} & $\mathrm{R}=\mathrm{H}$ & -51.4 & 92.5 & -24.5 & -57.6 & -7.3 & 38.9 & -53.6 & 54.9 \\
\hline & $\mathrm{CH}_{3}$ & -51.0 & 97.2 & -18.3 & -59.1 & -0.7 & 43.6 & -56.0 & 60.3 \\
\hline & $\mathrm{CH}_{2} \mathrm{Cl}$ & -53.6 & 100.0 & -19.9 & -58.4 & -2.5 & 42.3 & -55.0 & 58.7 \\
\hline \multirow[t]{3}{*}{$5 \mathrm{c}-e n d o$} & $\mathrm{R}=\mathrm{H}$ & -51.3 & 93.1 & -24.4 & -57.0 & -7.4 & 37.0 & -52.8 & 52.7 \\
\hline & $\mathrm{CH}_{3}$ & -55.4 & 99.6 & -22.7 & -56.7 & -5.8 & 38.2 & -51.7 & 53.6 \\
\hline & $\mathrm{CH}_{2} \mathrm{Cl}$ & -57.1 & 100.9 & -23.4 & -57.5 & -6.3 & 40.0 & -52.5 & 55.7 \\
\hline \multirow[t]{3}{*}{$5 c-e x o$} & $\mathrm{R}=\mathrm{H}$ & -51.6 & 92.3 & -24.7 & -57.8 & -7.5 & 36.5 & -53.6 & 52.5 \\
\hline & $\mathrm{CH}_{3}$ & -55.8 & 99.6 & -23.1 & -56.7 & -6.2 & 39.4 & -54.0 & 55.5 \\
\hline & $\mathrm{CH}_{2} \mathrm{Cl}$ & -57.1 & 101.5 & -23.4 & -56.9 & -6.4 & 37.5 & -50.6 & 52.6 \\
\hline
\end{tabular}

$\Delta \mathrm{H}_{f}=$ Heat of fomation. $\Delta \mathrm{H}, \Delta \mathrm{S}$, and $\Delta \mathrm{G}$ refer to reaction enthalpy, reaction entropy, and Gibbs free energy of reaction, respectively. $\Delta \mathrm{H}^{+}, \Delta \mathrm{S}^{\prime}$, and $\Delta \mathrm{G}^{\neq}$refer to activation enthalpy, activation entropy, and Gibbs free energy of activation. respectively."cali( $\left.\mathrm{K} \cdot \mathrm{mol}\right)$.

addition reaction of 1 with 1,3-cyclohexadiene at $298 \mathrm{~K}$.

Themodynamic parameters of endo and exo adduct were compared to see if there are stability differences that could result in endo/exo selectivity: The difference of Gibbs free energy of reaction, $\Delta \mathrm{G}$ (endo-exo) and the difference of

Table 5. Comparison of thermodynamic parameters between 1diene and 1-dienophile for the Diels-Alder reaction of 1 with 1,3cyclohexadiene in $\mathrm{kcal} / \mathrm{mol}$

\begin{tabular}{|c|c|c|c|c|c|}
\hline & $\Delta \Delta \mathrm{G}^{\mp}$ & $\Delta \Delta G$ & & $\Delta \Delta G^{2}$ & $\Delta \Delta \mathrm{G}$ \\
\hline$(4-e n d o)-(5-e n d o)$ & \multicolumn{5}{|c|}{$(4-e \times o)-(5-e \times o)$} \\
\hline $\mathrm{R}=\mathrm{H}$ & 0.5 & -1.2 & $\mathrm{R}=\mathrm{H}$ & -0.8 & -4.0 \\
\hline $\mathrm{CH}_{3}$ & -2.8 & -5.8 & $\mathrm{CH}_{3}$ & 2.8 & -1.1 \\
\hline $\mathrm{CH}_{2} \mathrm{Cl}$ & -1.0 & -5.1 & $\mathrm{CH}_{2} \mathrm{Cl}$ & 2.1 & -1.7 \\
\hline$(\mathbf{4 a}-e n d o)-(\mathbf{5} \mathbf{a}-e n d o)$ & \multicolumn{5}{|c|}{$(4 \mathbf{a}-\boldsymbol{e} \times o)-(5 \mathbf{a}-\boldsymbol{e} \times o)$} \\
\hline $\mathrm{R}=\mathrm{H}$ & 4.2 & -0.9 & $\mathrm{R}=\mathrm{H}$ & 1.4 & -1.3 \\
\hline $\mathrm{CH}_{3}$ & 3.6 & -2.5 & $\mathrm{CH}_{3}$ & 3.4 & -0.5 \\
\hline $\mathrm{CH}_{2} \mathrm{Cl}$ & 3.8 & -1.6 & $\mathrm{CH}_{2} \mathrm{Cl}$ & 3.4 & -0.9 \\
\hline$(\mathbf{4} \mathbf{b}-e n d o)-(\mathbf{5} \mathbf{b}-\epsilon \| t d o)$ & \multicolumn{5}{|c|}{$(\mathbf{4} \mathrm{b}-\mathrm{e} \times)-(5 \mathrm{~b}-\mathrm{e} \times)$} \\
\hline $\mathrm{R}=\mathrm{H}$ & -1.5 & -2.8 & $\mathrm{R}=\mathrm{H}$ & 2.3 & 1.6 \\
\hline $\mathrm{CH}_{3}$ & -1.9 & -5.3 & $\mathrm{CH}_{3}$ & -2.3 & -4.5 \\
\hline $\mathrm{CH}_{2} \mathrm{Cl}$ & -1.5 & -5.2 & $\mathrm{CH}_{2} \mathrm{Cl}$ & -0.8 & -3.0 \\
\hline$\left(4 \mathbf{c}-e^{-1} t d o\right)-\left(5 \mathbf{c}-e^{n} n d o\right)$ & \multicolumn{5}{|c|}{$(4 c-e x o)-(5 c-e x o)$} \\
\hline $\mathrm{R}=\mathrm{H}$ & 0.6 & -1.0 & $\mathrm{R}=\mathrm{H}$ & 3.0 & 0.3 \\
\hline $\mathrm{CH}_{3}$ & 1.3 & -2.2 & $\mathrm{CH}_{3}$ & 2.8 & 0.5 \\
\hline $\mathrm{CH}_{2} \mathrm{Cl}$ & -1.8 & -2.0 & $\mathrm{CH}_{2} \mathrm{Cl}$ & 5.6 & 1.4 \\
\hline
\end{tabular}

Gibbs free energy of activation, $\Delta \Delta \mathrm{G}^{+}$(endo-exo) reveal that 3-diene adducts are favourable for endo addition by 1.1-5.0 $\mathrm{kcal} / \mathrm{mol}$ and $0.0-4.8 \mathrm{kcal} / \mathrm{mol}$, respectively. 1-Dienophile adducts are favourable for endo addition by Gibbs free energies of activation of 1.3-3.7 kcal/mol, while Gibbs free energies of reaction do not show notable difference.

Themodynamic parameters of 1-diene and 1-dienophile were compared to see if there are stability differences that could result in 1-diene/1-dienophile selectivity (Table 5).

The differences of Gibbs free energy of reaction between 1-diene and 1-dienophile are favourable for 1-diene cycloaddition by $0.9-5.8 \mathrm{kcal}$ mol for endo adducts, but do not show consistancy for exo adducts within $0.5-4.5 \mathrm{kcal} / \mathrm{mol}$. And the differences of Gibbs free energy of activation, which range from $0.5 \mathrm{kcal} / \mathrm{mol}$ to $5.6 \mathrm{kcal} / \mathrm{mol}$, do not show consistency for both endo and exo adducts. These results are quite different from the results of the cycloaddition of 1 with cyclopentadiene which show reactions are thernodynamically controlled; the differences of Gibbs free energy of reaction are insignificant compared to those of 10.6-20.3 $\mathrm{kcal} / \mathrm{mol}$ for cycloaddition with cyclopentadiene. Potential energy diagrams for the cycloadditions of 1 with 1,3-cyclohexadiene are shown in Figure 3.

Table 6 shows thermodynamic parameters for the Cope rearrangement of endo adducts of 1 with 1,3 -cyclohexadiene.

Gibbs free energies of activation for the Cope rearrange- 


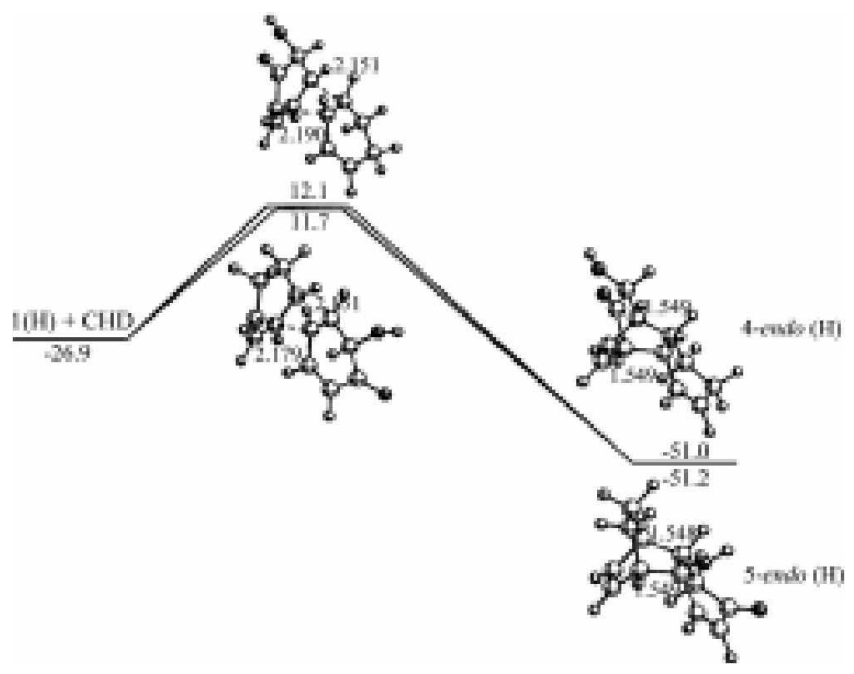

Figure 3. Potential energy $\left(\Delta \mathrm{H}_{\mathrm{f}}\right)$ diagrams for the cycloaddition in compound $1(\mathrm{R}=\mathrm{H})$ with 1,3-cyclohexadiene (CHD) calculated by the PM3 Hamiltonian. Energies and interatomic distances are given in kcal mol and angstrom, respectively.

ment of 1-dienophile endo adducts and 1-diene endo adducts are in the range of 55.7 to $63.3 \mathrm{kcal} / \mathrm{mol}$ and 60.2 to 64.3 , respectively. The values of Gibbs free energy of activation could be compared with those for the retro Diels-Alder reaction, which are in the range of 57.0 to $62.2 \mathrm{kcal} / \mathrm{mol}$ for 1-dienophile adducts and 61.7 to $65.5 \mathrm{kcal} / \mathrm{mol}$ for 1 -diene adducts. And Gibbs free energies of reaction for the Cope rearrangement of 1-dienophile endo adducts are small in the range of -0.9 to $-5.8 \mathrm{kcal} / \mathrm{mol} \mathrm{d}_{\mathrm{l}}$ and $\mathrm{d}_{2}$ values at ground state vary from $c a \cdot 1.55 \AA$ of normal C-C single bond distance to the range of $3.62 \AA-4.23 \AA$, and those values at transition state are in the range of $2.12 \AA-2.17 \AA$.

The observations in the cycloadditions of 1 with 1,3 cyclohexadiene, which gave both 1-diene adduct and 1dienophile adduct, might be explained by small differences of Gibbs free energy of reaction of $0.5-5.8 \mathrm{kcal} / \mathrm{mol}$. These insignificant differences of Gibbs free energy of reaction could be compared with those of $10.6-20.3 \mathrm{kcal} / \mathrm{mol}$ for cycloaddition of 1 with cyclopentadiene which gave solely 1-diene adduct.

Endoiexo selectivities of 1-diene adducts are favourable for endo adducts in terms of both Gibbs free energy of activation and Gibbs free energy of reaction, while the selectivity of 1-dienophile adducts are favourable for endo adducts in terms of Gibbs free energy of activation. This result coincides with experimental observation that endo adducts are formed in the cycloadditions of 1 with 1,3cyclohexadiene. $^{8 b}$ Nonetheless, Cope rearrangements of 1dienophile endo adduct to 1-diene endo adduct do not accord with the experimental observation; Although Cope rearrangements of 1-dienophile endo adduct to 1-diene endo adduct are slightly more favourable than those of 1-diene endo adduct to 1-dienophile endo adduct in terms of Gibbs free energies of reaction, they observed only Cope rearrangement of 1-diene endo adduct to 1-dienophile endo adduct.
Table 6. PM3-calculated themodynamic parameters for the Cope rearrangement of the adducts of 1 with 1,3-cyclohexadiene in $\mathrm{kcal} /$ $\mathrm{mol}$ at $298 \mathrm{~K}$

\begin{tabular}{|c|c|c|c|c|c|c|}
\hline Reactions & $\Delta \mathrm{H}$ & $\Delta S^{\prime \prime}$ & $\Delta \mathrm{G}$ & $\Delta \mathrm{H}^{\mp}$ & $\Delta S^{\nexists a}$ & $\Delta \mathrm{G}^{\mp}$ \\
\hline \multicolumn{7}{|l|}{ 5-endo to 4-endo } \\
\hline $\mathrm{R}=\mathrm{H}$ & 0.2 & 4.6 & -1.2 & 61.6 & 2.4 & 60.9 \\
\hline $\mathrm{CH}_{3}$ & -4.6 & 4.1 & -5.8 & 57.0 & 1.8 & 56.5 \\
\hline $\mathrm{CH}_{2} \mathrm{Cl}$ & -3.6 & 5.0 & -5.1 & 57.7 & 2.4 & 57.0 \\
\hline \multicolumn{7}{|l|}{$5 \mathrm{a}-e n d o$ to $4 \mathrm{a}-e n d o$} \\
\hline $\mathrm{R}=\mathrm{H}$ & 0.5 & 4.6 & -0.9 & 61.7 & 1.8 & 61.2 \\
\hline $\mathrm{CH}_{3}$ & -1.6 & 3.2 & -2.6 & 61.7 & 3.3 & 60.7 \\
\hline $\mathrm{CH}_{2} \mathrm{Cl}$ & -0.5 & 3.6 & -1.6 & 62.3 & 0.2 & 62.2 \\
\hline \multicolumn{7}{|l|}{$5 \mathrm{~b}-\mathrm{endo}$ to $4 \mathrm{~b}-\mathrm{endo}$} \\
\hline $\mathrm{R}=\mathrm{H}$ & -1.8 & 3.5 & -2.8 & 58.0 & 1.9 & 57.4 \\
\hline $\mathrm{CH}_{3}$ & -4.2 & 3.8 & -5.3 & 56.3 & 1.5 & 55.9 \\
\hline $\mathrm{CH}_{2} \mathrm{Cl}$ & -4.0 & 4.1 & -5.2 & 56.2 & 1.8 & 55.7 \\
\hline \multicolumn{7}{|l|}{$5 \mathrm{c}-e n d o$ to $4 \mathrm{c}-e n d o$} \\
\hline $\mathrm{R}=\mathrm{H}$ & 0.3 & 4.3 & -1.0 & 63.9 & 2.1 & 63.3 \\
\hline $\mathrm{CH}_{3}$ & -1.0 & 3.9 & -2.2 & 62.3 & 1.5 & 61.9 \\
\hline $\mathrm{CH}_{2} \mathrm{Cl}$ & -0.7 & 4.5 & -2.0 & 62.5 & 1.9 & 61.9 \\
\hline
\end{tabular}

"cali( $(\mathrm{K} \cdot \mathrm{mol})$

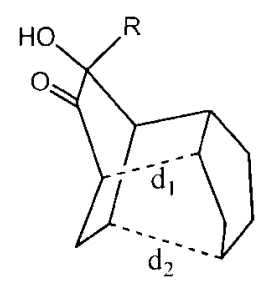

$\mathrm{TS}_{4-5}$

All above results imply that cycloadditions of 1 with cyclopentadiene are controlled thennodynamically to give only 1-diene adducts, while its reactions with 1,3-cyclohexadiene do not show 1-diene/1-dienophile selectivity due to similar stabilities of the two adducts. Themodynamic parameters show that endo adducts are more favourably forned in the cycloadditions of 1 with both cyclopentadiene and 1,3-cyclohexadiene, which coincides with experimental observations. Cope rearrangements of endo adducts are another avenue to convert between 1-diene and 1-dienophile.

Acknowledgment. We thank Professor V. K. Singh, Dept. of Chemistry, Indian Institute of Technology, for suggesting the problem. Partial cost of this research was defrayed by Andong National University, Korea.

\section{References}

1. Rigby, J. H. Comprehensive Organic Synthesis: Trost, B. M., Flemming. I.. Paquette, L. A., Eds.; Pergamon Press: Oxford, 1991; Vol. 5. pp 617-643.

2. (a) Lin. C. Y.; Ding. S. T. J. Org. Chem. 1992. 57, 4539. (b) Rigby. J. H.; Ateeg, H. S. J. Am. Chem. Soc. 1990, 112, 6442. (c) Wender; P. A. Correia, C. R. D. J. Am. Chem. Soc 1987, Ish, 2523. (d) Garst, M. E.: Roberts, V. A.: Prussin, C. J. Org. Chem. 1982, 47, 3969 .

3. (a) Haseltine, J. N.; Cabel, M. P.; Mantlo, N. B.; Iwasawa. N.; Yamasita. D. S.; Coleman. R. S.; Danishefsky. S.; Schulte, G. K. J. Am. Chem. Soc. 1991, 113, 3850 . (b) Corey, E. J.; Dittami, J. P. 
J. Am Chem Soc, 1985, 107, 256

4. (a) Singh, V. K: Thomas, B. J. Chem. Soc. Chem, Commun. 1992. 1211. (b) Singh. V. K.; Porinchu, M. J. Chem. Soc. Chem. Commun. 1993, 134. (c) Sing, V; Porinchu, M. Tetrahedron 1996, 52, 7087. (d) Singh. V.; Thomas, B. J. Org. Chem. 1997, 62, 5310. (e) Singh, V; Thomas, B. J. Indian Chem. Soc. 1998, 75, 640.

5. (a) Bratby, D. M.: Fray, G. I. J. Chem. Soc. (C) 1971, 970. (b) Bratby, D. M.; Chadwick. J. C.; Fray, G. I.; Saxton. R. G. Tetrahedrot 1977, 33, 1527 .

6. (a) Gesson, J. P.; Hervaud, L.; Mondon, M. Tetrahedron Lett. 1993, 34, 2941. (b) Bonnamne, V.; Bachmann. C.; Cousson, A.; Mondon, M.; Gesson, J. P. Tetrahedron 1999, 55, 433.

7. (a) Woodward, R. B.; Hoffman, R. Consertation of Orbital Symmetry; Academic Press: New York, 1972; pp 65-113. (b) Fleming. I. Frontier Orbitals and Organic Chemical Reactions; John Wiely and Sons: Chichester, 1978; Chapter 4, pp 86-181. (c)
Carruthers. W. Some Modem Methods of Organio Symthesis; Cambridge University Ptess: Cambridge, 1985; Chapter 3.pp 184-262.

8. (a) Singh, V. K.: Deota, P. T.: Bedekar, A. V. J. Chem. Soc. Perkin Trans. I 1992, 903. (b) Singh, V; Shama, U.; Prasanna, V.; Porinchu, M. Tetrahedron 1995, 51,6015.

9. March. J. Adranced Onganic Chemisty. 3rd ed.; John Wiley and Sons: New York, 1985; pp 745-758.

10. Stewart. J. J. P. J. Comput. Chem. 1989, 10. 209, 221.

11. Stewart, J. J. P. MOPAC 93; Fujitsu Limited: Tokyo, 1993

12. (a) Cerjan, C. J.: Miller, W. H. J. Chem Phys. 1981, 75, 2800. (b) Banerjee, A.: Adams, A.: Simons, J.: Shepard, R. J. Phys. Chem. 1985, 89.52.

13. (a) Gonzalez, C; Schlegel. H. B. I. Phys. Chem. 1989, 93. 2154. (b) Gonzalez. C; Schlegel. H. B. J. Pllys. Chem. 1990. 94.5523.

14. Mok, K. L.; Nye, M. J. J. Chem. Soc. Perkin I 1975, 1810. 